\title{
POLITIQUE DE PERFORMANCE ÉNERGÉTIQUE DES LOGEMENTS À BRUXELLES : UNE LOGIQUE INDUSTRIELLE STRUCTURELLEMENT INÉGALITAIRE
} Julie Neuwels

\author{
ERES | « Espaces et sociétés »
}

2017/3 n 170 | pages 73 à 90

ISSN 0014-0481

ISBN 9782749256023

Article disponible en ligne à l'adresse :

https://www.cairn.info/revue-espaces-et-societes-2017-3-page-73.htm

Distribution électronique Cairn.info pour ERES.

(C) ERES. Tous droits réservés pour tous pays.

La reproduction ou représentation de cet article, notamment par photocopie, n'est autorisée que dans les limites des conditions générales d'utilisation du site ou, le cas échéant, des conditions générales de la licence souscrite par votre établissement. Toute autre reproduction ou représentation, en tout ou partie, sous quelque forme et de quelque manière que ce soit, est interdite sauf accord préalable et écrit de l'éditeur, en dehors des cas prévus par la législation en vigueur en France. Il est précisé que son stockage dans une base de données est également interdit. 


\title{
coos
}

\section{Politique de performance énergétique des logements à Bruxelles : une logique industrielle structurellement inégalitaire}

\author{
Julie Neuwels
}

Cet et article propose une lecture des dimensions inégalitaires du logement énergétiquement performant établie à partir de l'analyse des fondements idéologiques des politiques publiques qui le formalisent : celle qui est relative à l'accès au logement, d'une part, et celle qui vise la performance énergétique des bâtiments, d'autre part. L'objectif est de mettre en évidence les leviers, les tensions et les compromis inhérents à la rencontre de ces deux grands pans des politiques de l'habiter. Cette analyse entend contribuer aux études abordant les difficiles conjugaisons entre droit au logement décent et performance énergétique des bâtiments, en s'attachant à leur signification plutôt qu'à leur (in)efficacité sociale.

Pour ce faire, la recherche s'appuie sur l'approche cognitive de la sociologie de l'action publique qui insiste sur l'importance des référentiels, soit des croyances partagées et politiquement construites de la réalité (Jobert et

\footnotetext{
Julie Neuwels, docteure en architecture et en urbanisme, collaboratrice scientifique à la faculté d'Architecture de l'université libre de Bruxelles et chercheuse-coordinatrice de projet au Brussels Studies Institute. jneuwels@ulb.ac.be
} 
Muller, 1987). Bien qu'appartenant à l'ordre de l'interprétation jusqu'à relever parfois du paralogisme, ces croyances se stabilisent et formatent les politiques publiques, en se matérialisant notamment dans des récits et des instruments d'action publique (Lascoumes et Le Galès, 2005). Ces instruments et ces récits sont représentatifs de la conception du rapport politique-société, du secteur concerné, des enjeux poursuivis et de leur hiérarchie. Leur analyse permet donc de mettre en lumière les confrontations et les ajustements entre référentiels.

Dans le cadre qui nous intéresse ici, l'approche par les référentiels est mise à profit pour questionner la notion de logement énergétiquement performant sous l'angle des transformations de la conception du logement minimum acceptable et de la protection de l'environnement, à partir de l'analyse des instruments (réglementations, plans stratégiques, etc.) et des récits (communiqués de presse, rapports de commissions parlementaires, littérature grise, etc.) qui structurent leur rencontre. Cette lecture s'établit à travers une attention particulière aux processus de « conversion-adaptation du "déjà là" de l'action publique » et aux rapports entre politiques globales et sectorielles (Lascoumes, 1996, p. 335). Ces appuis analytiques sont principalement issus de notre travail de thèse en architecture et en urbanisme qui, portant sur l'institutionnalisation bruxelloise de l'architecture durable, retrace la genèse des relations établies entre l'architecture, les idéologies développementalistes et l'action publique, des prémices de l'industrialisation à nos jours (Neuwels, 2015).

Le logement est en lui-même abordé comme un instrument de pensée et d'action (Lefebvre, 2000). Alors que le logement énergétiquement performant est généralement questionné en termes techniques (de performance) ou fonctionnels (d'accès), il s'agit par là d'insister sur le fait que cet objet d'étude est une construction sociale, qui procède d'agencements entre référentiels. Enfin, l'analyse porte sur le cas de la Région de Bruxelles-Capitale ${ }^{1}$, où les compétences ministérielles relatives à l'environnement et à l'énergie entre 2004 et 2014, et à la rénovation urbaine et au logement entre 2009 et 2014, ont été attribuées au parti politique écologiste Écolo. Ces attributions ont favorisé le développement d'une politique énergétique des bâtiments exigeante, a priori synergique et à teneur plus écologiste et sociale qu'économique.

L'article s'attarde dans un premier temps sur la manière dont les dimensions inégalitaires du logement performant sont abordées dans la littérature ainsi que sur les principales caractéristiques des politiques bruxelloises du logement et du bâtiment énergétiquement performant. Il décrit ensuite les deux grands points de vue qui se sont formalisés autour de la portée sociale de la dernière refonte réglementaire de la performance énergétique des bâtiments et

1. Une des trois régions composant la Belgique et regroupant dix-neuf communes, la Région de Bruxelles-Capitale, a été créée en 1989. Elle dispose d'un gouvernement et d'un parlement propres, dont les compétences fluctuent en fonction des remaniements du système institutionnel belge. 
le compromis technico-économique qui a permis d'apaiser les tensions à cet égard. Cette analyse met en évidence la prégnance d'une logique industrielle dans la politique énergétique des bâtiments à Bruxelles, dont la teneur est décrite et discutée à travers une comparaison avec la politique d'accès au logement, offrant une lecture plurielle des tensions inhérentes à leur rencontre. Il en ressort que les valeurs relatives à la minimisation des consommations énergétiques des bâtiments dominent celles du droit au logement dans l'institutionnalisation du logement performant à Bruxelles, l'inscrivant dans une logique industrielle structurellement inégalitaire. La question de l'équité sociale ne constitue plus un support structurant les politiques environnementales mais est abordée, au mieux, de manière palliative.

\section{LE LOGEMENT ÉNERGÉTIQUEMENT PERFORMANT AU CROISEMENT DE POLITIQUES DIVERSIFIÉES}

\section{Des asymétries de prises}

Les dimensions inégalitaires du logement énergétiquement performant mises en évidence dans la littérature peuvent être condensées en termes d' ' asymétries de prises » : des asymétries de capacité à faire face à l'augmentation et à l'évolution des contraintes, à intégrer les évolutions cognitives qu'elles suggèrent et à accéder aux ressources à disposition (Chateauraynaud, 2006). Les dimensions inégalitaires du logement énergétiquement performant agissent donc sur des registres subjectifs, liés à l'acceptabilité sociale, et objectifs, liés à l'accessibilité pratique.

Fonction de paramètres multiples et enchevêtrés (Hamman et al., 2014), les manquements de l'acceptabilité sociale sont régulièrement expliqués par le caractère techniciste des politiques énergétiques qui s'avèrent de ce fait peu adaptées à la réalité diversifiée et complexe des habitants (Zélem, 2010). La dimension inégalitaire qui en résulte est prégnante en ce qui concerne les informations relatives au logement performant. Prenant la forme de labels, de certificats, de bonnes pratiques habitantes, de fiches techniques ou encore de catalogues des aides disponibles, ces instruments sont particulièrement peu adaptés aux plus précarisés, sujets à une " analphabétisation technico-énergétique » (Grevisse, 2012, p. 64). Ces personnes éprouvent des difficultés à comprendre les labels et les certificats, les factures d'électricité, les manuels de fonctionnement et d'entretien des équipements, ou encore ils connaissent peu leurs droits et les aides à leur disposition (Devalière, 2009).

L'accessibilité pratique au logement énergétiquement performant est quant à elle souvent discutée en termes financiers. Les mécanismes de distribution des instruments économiques et fiscaux pour les travaux économiseurs d'énergie font l'objet d'une triple asymétrie de prises structurelle. Ces instruments ne sont accessibles qu'aux acteurs capables financièrement de construire 
ou de rénover un bien immobilier (Béal, 2011), non sans mener parfois à un effet d'aubaine, soit le recours à des aides publiques dans le cadre de travaux qui auraient de toute manière été effectués sans elles (Grosche et Vance, 2009). Ensuite, ces dispositifs ne permettent pas de résoudre le dilemme propriétaire-locataire qui veut que les propriétaires soient peu intéressés à investir pour améliorer les qualités thermiques du bâtiment qu'ils n'habitent pas (Lovell 2005). Enfin, les aides financières sont préférablement allouées à des projets considérés comme exemplaires, parfois exagérément dispendieux, au détriment « de l'intervention prioritaire sur les territoires aux séquelles écologiques et sociales » (Emelianoff, 2004, p. 35).

Ces phénomènes d'asymétrie sont essentiellement abordés par le biais de la mise à l'épreuve des politiques publiques. Ils apparaissent alors comme des problèmes, essentiellement fonctionnels, d'accès aux dispositifs. Les logiques à l'origine des dimensions inégalitaires du logement performant demeurent indirectement ou partiellement questionnées. Pourtant, l'institutionnalisation du logement énergétiquement performant se situe au croisement de politiques, celles du logement et de la performance énergétique des bâtiments, reposant sur des valeurs et poursuivant des objectifs distincts. Cette situation de croisement implique agencements et compositions significatifs, dont l'analyse permet de caractériser les dimensions inégalitaires du logement performant au-delà de l'accessibilité fonctionnelle.

\section{La politique bruxelloise du logement}

La politique du logement telle qu'elle est actuellement appliquée à Bruxelles vise le droit au logement décent et l'attractivité résidentielle de la classe moyenne. L'accès au logement décent s'est développé au tournant des XIX et $\mathrm{XX}^{\mathrm{e}}$ siècles, notamment sous l'impulsion de l'audience croissante des idéaux socialistes (Puissant, 2003). Il se formalise essentiellement par la construction et la mise en location de logements dits « sociaux » à bas loyers par des sociétés parapubliques et, depuis peu, par le secteur privé, à travers des agences immobilières sociales. Le droit au logement décent est également régulé par une série de normes minimales d'habitabilité, de sécurité et de salubrité, via le code du logement et le règlement régional d'urbanisme.

La politique d'attractivité résidentielle se développe depuis les années 1980. Elle cherche à faciliter l'accès à la propriété privée au travers d'incitations économiques accessibles aux propriétaires occupants et de la construction de logements " moyens » vendus ou loués en deçà des prix du marché et destinés à des personnes disposant de revenus correspondant à ceux de la classe moyenne. L'attractivité résidentielle s'inscrit dans une politique de revitalisation urbaine, mise en place pour lutter contre la paupérisation et la détérioration du cadre bâti résultant des politiques territoriales belges d'aprèsguerre, dominées par l'idéal du résidentiel périurbain et l'appréhension de 
Bruxelles comme une capitale administrative peu apte à l'habiter (Noel, 2009). Il s'agit également d'augmenter les finances régionales et communales, qui dépendent en partie de l'imposition des Bruxellois.

L'objectif d'attractivité domine la politique du logement à Bruxelles, non sans induire une augmentation des prix de l'immobilier dans les quartiers populaires et une répartition des aides financières qui s'établit au détriment des moins nantis (Van Criekingen, 2006). De fait, depuis la fin des années 1970, la hausse des prix de l'immobilier dépasse celle des revenus (Bernard et al., 2009). Selon le Baromètre social 2014 de l'Observatoire de la santé et du social de Bruxelles-Capitale, le prix moyen d'acquisition d'un logement à Bruxelles a doublé en dix ans, tandis que le loyer moyen corrigé de l'inflation a augmenté de $20 \%$ entre 2004 et 2013 (Englert et al., 2014). Simultanément, représentant $8 \%$ du parc résidentiel bruxellois, l'offre en logements sociaux s'avère deux fois moins élevée que la demande, le nombre de ménages en attente d'un logement social équivalant au parc existant (Dessouroux et Romainville, 2011). Ainsi, comme le relève la synthèse des états généraux de Bruxelles sur le logement du Brussels Studies, à la fin des années 2000, plus de la moitié des locataires dépensent entre $41 \%$ et $65 \%$ de leur revenu pour se loger, tandis que $20 \%$ à $30 \%$ des Bruxellois vivent dans une habitation ne répondant pas aux normes de confort et de salubrité (Bernard et al., 2009).

Cet état des lieux nous offre deux grands enseignements concernant le cas bruxellois. D'une part, la seule amélioration thermique du parc social y est insuffisante pour assurer l'équité d'accès à un logement performant. D'autre part, la politique de performance énergétique des bâtiments (PEB) s'y développe conjointement à une politique d'accès au logement elle-même sous tension, entre la nécessité d'assurer le droit au logement et celle de la revitalisation urbaine.

\section{La politique bruxelloise de performance énergétique des bâtiments}

Comme son nom l'indique, la PEB concerne l'ensemble du bâti, résidentiel et non résidentiel. Elle est par ailleurs intégrée au code bruxellois de l'air, du climat et de l'énergie, distinct des autres dispositifs régulant l'habiter. En même temps, le logement constitue un espace privilégié de mise en œuvre de la PEB bruxelloise.

L'institutionnalisation du logement performant s'est réellement développée à Bruxelles suite à l'attribution en 2004 à l'écologiste Evelyne Huytebroeck d'un ministère régional de l'Environnement et de l'Énergie, complété cinq ans plus tard par la compétence relative à la rénovation urbaine. Dans un premier temps, la PEB s'est principalement constituée à travers une logique de responsabilisation par le développement d'informations, de diffusion de bonnes pratiques, de primes et de prêts à taux réduits, de formations, ou encore d'appels à projets. Dans un second temps, sous l'impulsion d'une directive 
européenne visant la « consommation d'énergie quasi nulle » des bâtiments ${ }^{2}$, la PEB bruxelloise a connu un durcissement réglementaire majeur. Devançant les délais et les minima de la directive, la législation bruxelloise impose que tout logement neuf respecte des critères proches de la construction passive depuis 2010 et 2015 respectivement pour les secteurs publics et privés ${ }^{3}$ (PEB, 2015). Transitoire, cette réglementation devrait aboutir au « passif » stricto sensu puis au «zéro énergie » ${ }^{4}$ en 2019 pour le parc public et 2021 pour le parc privé. Les rénovations de logements sont quant à elles soumises à des obligations moindres, définies en fonction des travaux réalisés.

Un logement est dit « passif » lorsque l'énergie utilisée pour le chauffage est issue presque exclusivement des occupants, des appareils (éclairage, électroménager, etc.) et du rayonnement solaire. Ce haut niveau de performance est notamment atteint grâce à une isolation thermique poussée, à une haute étanchéité à l'air et à un système de ventilation mécanique contrôlée, généralement à double flux, qui assure l'échange de chaleur ou de fraîcheur entre l'air entrant et l'air sortant. Le choix du passif ne constitue donc pas un simple durcissement réglementaire : il s'agit d'imposer ce qui est généralement considéré comme la meilleure performance énergétique possible sans recours aux équipements produisant de l'énergie renouvelable. Le « zéro énergie » introduira l'obligation de mise en œuvre de tels équipements. Les exigences sont telles que certaines unités habitables présentant une situation thermique défavorable, tel un manque de compacité ou d'ensoleillement, ne peuvent techniquement pas respecter la réglementation, obligeant le gouvernement bruxellois à prévoir un système de dérogation automatique. Autrement dit, la référence au passif et au zéro énergie implique que les réglementations PEB n'imposent plus un minimum acceptable mais bien un « maximum espéré » (Neuwels, 2015).

\section{UN COMPROMIS TECHNICO-ÉCONOMIQUE}

\section{Réglementation asociale versus mesure d'intérêt général}

Loin d'être particulier à Bruxelles, l'avènement réglementaire et sociocognitif du logement performant est généralement expliqué par la « carbonisation » du développement durable (Zaccai, 2011), c'est-à-dire la prépondérance de la lutte contre le réchauffement climatique dans les

2. Directive 2010/31/UE du Parlement européen et du Conseil, du 19 mai 2010, sur la performance énergétique des bâtiments (refonte).

3. Arrêté du gouvernement de la Région de Bruxelles-Capitale (ARGB) du 4 juin 2009 fixant les normes énergétiques applicables aux projets subventionnés de travaux visant à L'URE (utilisation rationnelle de l'énergie) dans les bâtiments appartenant aux communes et CPAS (centres publics d'action social) ; ARGB du 21 février 2013 modifiant L'ARGB du 21 décembre 2007 déterminant des exigences en matière de performance énergétique et de climat intérieur des bâtiments.

4. Code bruxellois de l'air, du climat et de l'énergie, Livre 2, Titre 2, Art. 2.2.3. 
politiques environnementales depuis le protocole de Kyoto (1997), qui favorise la recherche de minimisation des émissions de gaz à effet de serre et, par extension, des consommations énergétiques liées à l'usage des bâtiments. Si la présence des écologistes au sein du gouvernement bruxellois a renforcé le poids de la carbonisation dans la régulation du logement performant, elle ne suffit pas pour expliquer l'imposition d'un « maximum espéré ». Le gouvernement bruxellois était composé de cinq (2004-2009) à six (2009-2012) formations politiques moins sensibles aux enjeux environnementaux, que les écologistes ont dû convaincre et avec lesquelles ils ont dû composer. En particulier, le choix du passif a induit des tensions quant aux effets sociaux de ses surcoûts.

Effectivement, la réglementation PEB 2015 n'a pas été déterminée en fonction du critère financier alors que la directive européenne à son origine suggère et permet de considérer le rapport coût-efficacité, soit les retours sur investissement, pour assurer la rentabilité à terme des travaux économiseurs d'énergie. Le cas bruxellois se distingue ainsi par une logique de coût global partagé, qui intègre certains impacts des bâtiments sur l'environnement naturel au-delà des seuls coûts et bénéfices financiers des maîtres d'ouvrage et des utilisateurs $^{5}$ (MIQCP, 2006).

Les surcoûts de la PEB 2015 sont généralement estimés entre $10 \%$ et $20 \%$, voire $30 \%$ selon des architectes interviewés, par rapport aux constructions respectant la réglementation antérieure ${ }^{6}$. Certains acteurs, dont des membres du Parti socialiste, estiment que ces surcoûts sont excessifs car non rentabilisés à terme par les gains d'énergie. Le choix du passif a ainsi été qualifié d' « asocial », d' ' irrationnel » et d' « élitiste ». Selon eux, le " (très) basse énergie » aurait été davantage pertinent car socio-économiquement plus équilibré. Les promoteurs de la PEB 2015, qui sont essentiellement des écologistes et des associations de promotion du passif, estiment au contraire que la construction passive est une mesure d'intérêt général, une exigence environnementale dont la pertinence ne peut être subordonnée à l'argument financier. De leur point de vue, l'argument du surcoût repose sur des rationalités obsolètes, peu capables de considérer les apports environnementaux et, par extension, sociaux de ce choix politique. La suite de l'article décrit ce rapprochement entre le passif et l'intérêt général.

5. Une dérogation est possible lorsque le respect des exigences PEB s'avère « économiquement irréalisable » soit, dans la pratique, si les travaux sont trois fois plus chers que des travaux de même nature dans un autre bâtiment.

6. Les surcoûts immédiats de la construction passive varient selon le contexte (orientation, mitoyenneté, etc.), la capacité des concepteurs à équilibrer les investissements ou encore les ajustements du marché. Le retour sur investissement est complexe à évaluer à cause de l'imprévisibilité des évolutions du prix de l'énergie et des comportements habitants. 


\section{Les croissances économique et technicienne comme leviers d'acceptation}

Pour apaiser ces tensions, les défenseurs du choix du passif ont bénéficié de deux grandes fenêtres d'opportunité, technique et économique. Premièrement, le secteur de la construction se prête relativement bien à la mise en œuvre de politiques de réduction des émissions carbone qui, cela fut régulièrement rappelé par les écologistes, sont soumises à obligation par l'Union européenne. D'une part, il existe des solutions techniques et technologiques permettant d'améliorer drastiquement la performance énergétique des bâtiments et donc d'améliorer le bilan carbone de la région. D'autre part, ces solutions s'avèrent relativement peu coûteuses à mettre en œuvre comparativement à d'autres domaines d'action car elles nécessitent une refonte des processus de conception et de construction architecturales (Carassus, 2007), et non pas une refonte des processus industriels.

Deuxièmement, l'adoption de la PEB 2015 a été grandement facilitée par l'idée qu'elle profiterait à la croissance économique et ce, malgré - voire grâce à - l'approche en termes de coût global partagé (Genard et Neuwels, 2016). En effet, l'amélioration de la performance énergétique des bâtiments est apparue comme un moyen d'assurer la dynamique économique du secteur de la construction par le développement de nouvelles filières et d'emplois, dont certains seraient accessibles à des personnes peu qualifiées. En outre, l'adoption d'une politique énergétique ambitieuse est utilisée pour améliorer l'attractivité, notamment résidentielle, de Bruxelles, en construisant une « marque de fabrique $»^{7}$, celle d'une ville $\left\langle\right.$ verte $»$ et $\left\langle\right.$ exemplaire $»^{8}$.

Ces agencements ne sont pas exceptionnels. En Belgique, les politiques liées au secteur de la construction occupent une place importante tant dans les stratégies de lutte contre le chômage que dans les stratégies d'attractivité, respectivement depuis les années 1950 et 1990. Plus largement, l'articulation des politiques environnementales avec le référentiel de marché est un lieu commun, voire un passage obligé (Rumpala, 2003). Contrairement à d'autres domaines d'action, cet agencement n'empêche pas la mise en œuvre d'une politique environnementale exigeante dans le secteur bruxellois de la construction. Au contraire, il a constitué un véritable levier permettant de légitimer une réglementation imposant la meilleure performance possible.

7. Plan de développement international de Bruxelles : schéma de base, Région de Bruxelles-Capitale, p. 70.

8. Projet de Plan régional de développement durable de la Région de Bruxelles-Capitale, p. 12, p. 150 et p. 264. 


\section{UNE LOGIQUE INDUSTRIELLE}

En même temps, l'appui sur ces deux fenêtres d'opportunité inscrit le logement énergétiquement performant dans une logique industrielle, dans le sens où les problèmes, les enjeux et les solutions sont pensés à partir des opportunités de croissance économique et des possibilités techniques, qui sont eux-mêmes fonction de la performance des équipements et des matériaux mais aussi des indicateurs énergétiques (Neuwels, 2015). Héritage des idéaux modernistes, la politique du droit au logement décent repose également en partie sur une logique industrielle (Champy, 2009) mais ses modalités, ses objectifs et ses valeurs diffèrent de ceux de la politique énergétique des bâtiments (figure 1). Une lecture comparée permet de caractériser la teneur de ces deux logiques et donc, leur difficile rencontre.

\begin{tabular}{|c|c|c|}
\hline Logique industrielle & $\begin{array}{l}\text { Politique du logement } \\
\text { (modernité) }\end{array}$ & $\begin{array}{l}\text { Politique énergétique des bâtiments } \\
\text { (modernisation écologique) }\end{array}$ \\
\hline $\begin{array}{l}\text { Recherche de } \\
\text { performance }\end{array}$ & Économique et sociale & Économique et environnementale \\
\hline Problématisation & Sanitaire et morale & Environnementale et morale \\
\hline \multirow[t]{2}{*}{ Horizon politique } & Sanitaire & Risque \\
\hline & Lutte contre le mal-logement & $\begin{array}{l}\text { Minimisation des impacts } \\
\text { environnementaux }\end{array}$ \\
\hline Récupération partielle & De la critique sociale & De la critique écologique \\
\hline \multirow[t]{2}{*}{ Objectifs globaux } & $\begin{array}{l}\text { Minimisation des coûts de } \\
\text { construction }\end{array}$ & $\begin{array}{l}\text { Relance économique et transition de } \\
\text { l'économie dans une logique verte }\end{array}$ \\
\hline & $\begin{array}{l}\text { Logement comme remède aux } \\
\text { dégradations des conditions } \\
\text { d'habiter }\end{array}$ & $\begin{array}{l}\text { Logement comme remède aux } \\
\text { dégradations environnementales }\end{array}$ \\
\hline \multirow[t]{2}{*}{ Solutions } & Standardisation et préfabrication & Performance environnementale \\
\hline & Postulats spatialistes & Éco-innovation, artefacts techniques \\
\hline \multirow[t]{3}{*}{ Cibles d'action } & Conditions d'habiter & Modes d'habiter \\
\hline & Classes sociales & Individu \\
\hline & Responsabilité des pouvoirs publics & Responsabilité des acteurs en situation \\
\hline
\end{tabular}

Figure 1 - Lecture comparée des principales caractéristiques des logiques industrielles en œuvre dans les politiques du logement et de performance énergétique des bâtiments.

(Source : enquête de terrain) 


\section{La question financière}

La logique industrielle du droit au logement vise la performance économique et sociale. Il s'agit de tirer profit du progrès technique pour minimiser les coûts de la construction et ainsi offrir un logement convenable au plus grand nombre. Cette logique est pensée en termes de minimisation de la maind'œuvre, de l'ornementation, des matériaux utilisés, et de standardisation des éléments constructifs et des bâtiments (Champy, 2009). Elle a notamment favorisé l'élaboration de plans standardisés de logements sociaux construits à Bruxelles dans la seconde moitié du XXe siècle (Aron, 1995).

La logique industrielle du logement énergétiquement efficient vise quant à elle la performance environnementale et économique. Nous l'avons vu, il s'agit de tirer profit de la croissance technicienne et économique pour mettre en œuvre une politique énergétique ambitieuse. Et, inversement, il s'agit de tirer des bénéfices financiers de la haute performance énergétique des bâtiments, en sachant que ceux-ci sont moins appréciés au regard des supposés retours sur investissement revenant aux habitants qu'à l'échelle de la relance économique supposée « verte » de la région. Le volet social est abordé au second plan, au travers des promesses de création d'emplois et de dynamisme économique du secteur de la construction. Cette hiérarchie de valeurs se répercute notamment sur la portée sociale des instruments visant à améliorer la capacité financière des potentiels maîtres de l'ouvrage.

Un argument couramment avancé par les promoteurs de la PEB 2015 pour apaiser les critiques relatives aux surcoûts est la mise à disposition d'aides financières, tels les "primes énergie » et «prêts verts » à taux zéro. Plus que l'atténuation des surcoûts, ces instruments cherchent aussi à dynamiser le secteur de la construction, en incitant les rénovations thermiques et en développant des emplois. Les incitations financières sont soumises à obligation de recours à des praticiens, à des équipements et à des travaux dûment certifiés, ce qui limite l'intérêt du faire soi-même a priori plus adapté aux personnes à faibles capacités financières. Il implique également de nouveaux coûts liés aux recours à des auditeurs et des certificateurs. Soit un ensemble de dispositifs techniques que les pouvoirs publics compétents présentent également comme des moyens leur assurant un regard sur les performances des travaux économiseurs d'énergie.

\section{Un effet d'aubaine considéré comme peu problématique}

Présent dès les premières heures de l'industrialisation, le souci pour l'environnement dans la régulation de l'habiter s'inscrivait jusqu'à il y a peu dans des rapports utilitariste et anthropocentriste à la nature (Neuwels, 2015). Ceux-ci ont favorisé une définition des problèmes axée sur le mal-logement et le cadre de vie, et une définition des moyens en termes esthétiques et formels. 
Entre autres, La charte d'Athènes, traité majeur du modernisme, dénonçait la perte des «conditions de nature », du « soleil», de l'« espace» et de la « verdure » définis comme des « nourritures fondamentales, d'ordre aussi bien psychologique que physiologique $»^{9}$. Dans la lignée hygiéniste, les relations entre l'homme et la nature étaient pensées sous l'angle de la pénétration du soleil, des flux d'air et des espaces verts présentés comme le « poumon de la ville » protégeant des « malaises » et des « maladies ${ }^{10}$. L'amélioration des conditions d'habiter de l'homme devait pallier les dommages causés par l'industrialisation, menant certains environnementalistes de l'époque à voir dans le modèle moderniste un remède aux destructions environnementales (Anker, 2010).

À Bruxelles, cette appréhension utilitariste et anthropocentriste de l'environnement s'est d'abord formalisée dans les politiques d'embellissement de la ville (construction de bâtiments néoclassiques, percement de grands boulevards, etc.) puis, à partir des années 1970, dans les politiques de rénovation (espaces verts, lutte contre les immeubles à l'abandon, rénovation de façades, etc.). Actuellement, symptomatique d'un glissement d'un horizon sanitaire à un horizon du risque, c'est moins la lutte contre l'insalubrité que la survie de l'homme qui domine la perception du rapport à la nature dans les politiques publiques. Le logement apparaît moins comme un remède aux dégradations des conditions d'habiter, que comme un remède aux dégradations environnementales liées au réchauffement climatique.

Dès lors, les effets d'aubaine ne sont pas considérés comme étant réellement problématiques à Bruxelles. Il y a bien eu quelques adaptations visant à limiter le phénomène. Les montants octroyés des " primes énergie » varient selon les revenus au profit des moins nantis et sont revus annuellement pour limiter les excès. Ces révisions sont toutefois restreintes car les effets d'aubaine sont perçus comme un moyen d'améliorer le bilan environnemental global de la région et, par extension, un phénomène profitant in fine à l'ensemble des Bruxellois ${ }^{11}$. L'équité des mécanismes de distribution est donc peu questionnée. Pourtant, les primes sont partiellement financées par l'ensemble des Bruxellois à travers un prélèvement automatique sur les factures de gaz et d'électricité. Proportionnellement, ce prélèvement pèse plus lourd sur le portefeuille des plus précarisés, tandis que les bénéficiaires des primes y contribuent moins, suite à la baisse de leur consommation résultant des travaux économiseurs d'énergie.

9. Le Corbusier, 1957 [1942], La Charte d'Athènes, Paris, Éditions de Minuit, p. 36.

10. Ibid., p. 34.

11. Commission de l'environnement, de la conservation de la nature, de la politique de l'eau et de l'énergie du 10 janvier 2012, Parlement de la Région de Bruxelles-Capitale, p. 28-37. 


\section{Le dilemme propriétaire-locataire}

Les politiques de performance énergétique des bâtiments sont principalement abordées en termes de "modes d'habiter », tandis que les politiques du logement se réfèrent aux « conditions d'habiter ». La référence aux conditions d'habiter sous-entend la résolution des disparités sociales et raisonne en termes de classes sociales. Elle sous-entend également la nécessité d'une prise en charge publique visant, à Bruxelles, les plus démunis dans le cadre du droit au logement et la classe moyenne dans le cadre des politiques d'attractivité urbaine, notamment par la production publique de logements. La référence aux modes d'habiter revêt une dimension plus individualiste, insistant sur la responsabilité des habitants. Objet d'une appréhension mécanique des comportements, l'habitant est abordé comme un consommateur responsable, supposément informé qui, par ses choix d'habiter, peut faire évoluer l'offre du logement. Les pouvoirs publics sont alors relégués à un rôle d'informateur, de facilitateur et de contrôleur.

Cette approche pose en particulier problème pour les logements en location, représentant environ $60 \%$ du parc résidentiel bruxellois, qui sont soumis au dilemme propriétaire-locataire. Ne profitant pas directement des retours sur investissements, les propriétaires se montrent peu intéressés à effectuer des travaux économiseurs d'énergie du ou des bâtiments qu'ils n'habitent pas (Lovell, 2005). Diverses mesures ont été prises pour résorber ce dilemme. Par exemple, dans un but d'autorégulation par le marché, un certificat de performance énergétique des bâtiments a été rendu obligatoire pour toute transaction immobilière d'un logement. Encore, dans un objectif de facilitation, la Région bruxelloise développe un système de charge d'investissement économiseur d'énergie. Actuellement à l'étude, ce projet consiste en l'élaboration d'un outil évaluant la juste répercussion des coûts des travaux économiseurs d'énergie sur les loyers. L'objectif est d'instaurer une réversibilité financière de l'investissement desdits travaux à charge du propriétaire vers le locataire, déterminée en fonction de leur montant, de la durée du paiement et des économies d'énergie théoriques.

Les solutions proposées suivent donc une logique de responsabilité individuelle, et sont pensées avant tout en termes de performance technique plutôt que d'usage. La rénovation thermique du parc résidentiel s'établit en fonction du bon vouloir des propriétaires, mécanique par ailleurs extrapolée par l'idéal de la propriété privée particulièrement ancrée en Belgique. Autrement dit, la question d'une prise en charge directe par les pouvoirs publics de la rénovation thermique des logements privés ne se pose pas. 


\section{Le cas de l'insalubrité énergétique}

Au-delà des asymétries de prises, s'esquisse le spectre d'un clivage social organisé en fonction des qualités énergétiques des logements (Grevisse, 2012). Ce clivage s'avère d'autant plus problématique que le phénomène de précarité énergétique, qui touche les personnes qui allouent plus de $10 \%$ de leurs revenus à leurs consommations énergétiques, croît à Bruxelles. Le rapport du régulateur bruxellois pour l'énergie traitant des données de 2012 (Brugel, 2013) fait état d'une augmentation de $50 \%$ en cinq ans d'installation de systèmes limitant la puissance des compteurs électriques et donc l'accès à l'électricité, d'une augmentation des dettes auprès des fournisseurs d'énergie pour un montant global d'environ 55 millions d'euros, d'une augmentation des demandes de résiliation de contrat par des fournisseurs auprès de la justice de paix qui peuvent mener à la fermeture des compteurs et qui ont concerné plus de 58000 ménages bruxellois en 2012, ou encore d'une augmentation du recours au statut de client protégé empêchant la résiliation des contrats par les fournisseurs.

La lutte contre la précarité énergétique consistait jusqu'à il y a peu en l'instauration de droits assurant un accès minimum à l'énergie. Avec la carbonisation du développement durable, elle englobe une dimension architecturée. La performance énergétique des bâtiments était notamment définie comme partie intégrante de la qualité des logements dans le projet de Plan régional de développement durable de $2013^{12}$. Selon cette logique, le deuxième Plan d'action en efficacité énergétique de la Région bruxelloise proposait d'imposer une performance minimale pour les logements en location par l'introduction du concept d' « insalubrité énergétique » dans le code du logement ${ }^{13}$, ce qui constituerait une obligation indirecte de rénovation thermique visant explicitement le dilemme propriétaire-locataire. Ce projet a été abandonné car il risque d'entraîner une diminution des logements salubres disponibles à la location et, par contagion, de mener à une augmentation des loyers dépassant les bénéfices de la diminution des charges énergétiques.

Ces effets pourraient être contenus par un encadrement contraignant des loyers, qui fait défaut à Bruxelles depuis 1984. Mis en œuvre en 1975 à l'échelle de la Belgique, l'encadrement des loyers avait favorisé en dix ans une baisse de 17,27\% des loyers par rapport à l'évolution des prix à la consommation (François et Laconte, 1984). En même temps, cette politique fut accusée d'avoir favorisé le désintérêt pour l'investissement immobilier et l'importante récession que connut le secteur de la construction, alors affecté par les crises

12. Projet de Plan régional de développement durable (2013), politiques sectorielles au service du développement durable, chapitre $1:$ « Des logements de qualité pour tous », p. 121-136.

13. Deuxième Plan d'action en efficacité énergétique de la Région bruxelloise, Mesure B.9 du secteur du bâtiment. 
économiques de l'époque. Depuis lors, l'encadrement des loyers est considéré comme une mesure socialement peu efficiente, notamment au regard de ses supposées répercutions sur les emplois liés au secteur de la construction.

Autrement dit, si dans le cadre de la politique du logement, les normes d'habitabilité sont considérées comme des mesures d'intérêt général, le cas de l'insalubrité énergétique est plus complexe : au vu de l'ampleur des coûts immédiats des travaux économiseurs d'énergie, il nécessiterait un encadrement des loyers qui entre en concurrence avec les stratégies de croissance économique, via les secteurs de la construction et de l'immobilier, établies par les pouvoirs publics bruxellois.

\section{CONCLUSION}

L'institutionnalisation du logement énergétiquement performant se situe au croisement de la politique du logement, traditionnellement majoritairement portée par des partis socialistes, et celle de la performance énergétique des bâtiments, développée sous l'impulsion d'un parti et des mouvements écologistes. Ces deux politiques et leurs acteurs présentent des rapports au social, à l'économie et à la nature distincts. Dans le cadre de la politique du logement, concomitamment à la recherche de minimisation des coûts de construction, l'environnement est abordé comme un moyen permettant d'améliorer les conditions d'habiter, de la salubrité du logement au cadre de vie. Non sans négliger une série de problématiques environnementales dont, jusqu'à récemment, celles qui sont liées à l'énergie. Inversement, dans le cadre de la politique énergétique des bâtiments, par sa rigueur réglementaire, le logement apparaît comme un moyen permettant de pallier voire de résoudre certaines dégradations environnementales. À Bruxelles, ce raisonnement est poussé à son paroxysme, jusqu'à imposer un " maximum espéré », exemple rare de politique de développement durable fort où la primauté de la valeur environnementale n'est pas subordonnée à l'argument des surcoûts incombant aux maîtres d'ouvrage (Neuwels, 2015 ; Genard et Neuwels, 2016). Mais, non sans paradoxe, cette politique forte a pu être mise en œuvre grâce à un argumentaire construit autour des idées de performance technique et de croissance économique, au risque de réduire la perception du logement énergétiquement performant à un problème technique et à une opportunité économique.

Force est de constater que les valeurs relatives à la consommation énergétique des bâtiments dominent celles du droit au logement dans la définition des instruments réglementaires et incitatifs qui formalisent le logement performant, l'inscrivant dans une logique industrielle structurellement inégalitaire. Ce qui ne fait pas écho à cette logique est peu ou pas questionné. Le rapport au social est indirectement abordé, entre autres, à travers la lutte contre le réchauffement climatique, la diminution des charges énergétiques ou encore la création d'emplois. Lorsqu'elle n'est tout simplement pas ignorée, la dimension inégalitaire 
du logement énergétiquement performant est traitée de manière palliative, par l'élaboration ou l'adaptation d'instruments censés résorber certains effets secondaires conflictuels.

En même temps, dans un incontestable contexte d'urgence de réduction des émissions carbone et de la précarité énergétique, les bénéfices du logement énergétiquement performant ne peuvent être ignorés. Ce n'est donc pas l'ampleur des exigences de la réglementation énergétique bruxelloise qui pose question mais bien la relégation du registre social au second plan. À cet égard, le logement performant apparait finalement comme le prolongement d'une inscription des politiques de l'habiter dans des logiques économiques mises en œuvre depuis plusieurs années et qui, à Bruxelles et ailleurs, favorisent notamment l'attractivité résidentielle et la concurrence interurbaine. Sous cet angle, les asymétries de prises du logement performant apparaissent moins comme un problème fonctionnel d'accès aux dispositifs de la politique de performance énergétique des bâtiments que comme un symptôme, parmi d'autres, d'un droit au logement décent déficient et en perte de vitesse. Il n'en demeure pas moins que le risque existe de voir les pouvoirs publics considérer ces asymétries comme un effet secondaire inévitable et non comme la marque d'un dysfonctionnement social structurel. De même, le risque existe de voir ce dysfonctionnement croître sous l'effet des inflexions de la récente politique énergétique telle que l'amoindrissement de l'autonomie par le recours croissant à la certification, l'individualisation de la transition énergétique des logements par l'approche centrée sur la figure du propriétaire, ou encore la confusion entre le logement et le produit de consommation favorisée par le déploiement de labels.

\section{RÉFÉRENCES BIBLIOGRAPHIQUES}

Anker Peder, 2010, From Bauhaus to Ecohouse. A History of Ecological Design, Baton Rouge, Louisiana State University Press.

ARON Jacques, 1995, La Cambre et l'architecture: un regard sur le Bauhaus belge, Bruxelles, Mardaga.

BÉAL Vincent, 2011, «Ville durable et justice sociale. Ce que le développement durable nous dit de la production de l'urbain ", Le développement durable changera-t-il la ville ? Le regard des sciences sociales, V. Béal, M. Gauthier et G. Pinson éd., Saint-Étienne, Publications de l'université de Saint-Étienne, p. 239-259.

BERNARD Nicolas, ZIMMER Pol et SURKIN Johan, 2009, « Le logement, la maîtrise foncière et l'espace public » [en ligne], Brussels Studies, Note de synthèse $\mathrm{n}^{\circ} 6$, [URL : http://brussels.revues.org/pdf/924], consulté le 17 février 2017.

BRUGEL, 2013, Rapport sur l'exécution de ses obligations, sur l'évolution du marché régional de l'électricité et du gaz et sur le respect des obligations, les mesures prises et les résultats obtenus de service public par le gestionnaire du réseau de distribution et les fournisseurs et spécialement en matière des droits des consommateurs résidentiels, Bruxelles, Rapport annuel pour la Commission de régulation de l'énergie en Région de Bruxelles-Capitale. 
CARASSUS Jean, 2007, « Trois modèles de maîtrise de l'énergie dans les bâtiments. Une comparaison internationale », Les annales de la recherche urbaine, $\mathrm{n}^{\circ} 103$, p. 123-133.

CHAMPY Florent, 2009, « L'engagement des professionnels comme conséquence de tensions consubstantielles à leur pratique : l'architecture moderne entre les deux guerres ", Sociétés contemporaines, ${ }^{\circ}$ 73, p. 97-119.

Chateauraynaud Francis, 2006, Les asymétries de prises. Des formes de pouvoir dans un monde en réseaux, Documents du GSPR, Paris, Éditions de L'EHESS.

Dessouroux Christian et RomainVILle Alice, 2011, « La production de logements en Belgique et à Bruxelles. Acteurs, dynamiques, géographie » [en ligne], Echogéo, $n^{\circ} 15$, [URL : https://echogeo.revues.org/12279], consulté le 2 novembre 2016.

DEVALIÈRE Isolde, 2009, « De l'inconfort thermique à la précarité énergétique, profils et pratiques des ménages pauvres ", Informations sociales, $\mathrm{n}^{\circ} 155$, p. 90-98.

EMELIANOFF Cyria, 2004, « Les villes européennes face au développement durable : une floraison d'initiatives sur fond de désengagement politique », Cahiers $d u$ proses, $\mathrm{n}^{\circ} 8$, p. 1-23.

Englert Marion, LuYTEn Sarah, Hercot David et Mazina Déogratias, 2014, Baromètre social 2014, Observatoire de la santé et du social de Bruxelles-Capitale.

FRANÇOIS André et LACONTE Pierre, 1984, «L'investissement immobilier en Belgique : 10 ans de politique », Courrier hebdomadaire du CRISP, $\mathrm{n}^{\circ} 1048$.

GENARD Jean-Louis et NEUWELS Julie, 2016, « Le développement durable comme objet de transactions » [en ligne], Sociologies, [URL : http://sociologies.revues. org/5378], consulté le 2 novembre 2016.

GREVISSE François, 2012, Les impacts sociaux des nouvelles règlementations relatives à la performance énergétique des bâtiments en Belgique, Bruxelles, Étude exploratoire pour la Fondation Roi Baudouin.

GRosche Peter et VANCE Colin, 2009, « Willingness to pay for energy conservation and free-ridership on subsidization : evidence from Germany », Energy Journal, no 2, p. 135-154.

HAmman Philippe, Frank Cécile et MANGOLD Marie, 2014, « Les trajectoires de conversion écologique face aux enjeux économiques et sociaux du "logement durable” en France » [en ligne], Vertigo, [URL : http://vertigo.revues.org/15018], consulté le 2 novembre 2016.

JoBert Bruno et MulLer Pierre, 1987, L'état en action, Paris, Presses universitaires de France.

LASCOUMES Pierre, 1996, « Rendre gouvernable : de la "traduction" au "transcodage". L'analyse des processus de changement dans les réseaux d'action publique », La gouvernementalité, Curapp éd., Paris, Presses Universitaires de France, p. 325-338.

LASCOUMES Pierre et LE GALÈS Patrick, 2005, « Introduction. L'action publique saisie par les instruments », Gouverner par les instruments, P. Lascoumes et P. Le Galès éd., Paris, Presses de Science Po, p. 11-44.

LeFEBVRE Henri, 2000 [1974], La production de l'espace, Paris, Anthropos.

LOVELL Heather, 2005, « Supply and demand for low energy housing in the UK : insights from a science and technology studies approach », Housing Studies, $\mathrm{n}^{\circ} 5$, p. 815-829. 
MIQCP, 2006, Ouvrages publics et coût global : une approche actuelle pour les constructions publiques, Paris, Mission interministérielle pour la qualité des constructions publiques.

Neuwels Julie, 2015, Architecture, développement et action publique : conjugaison en mutation dans un contexte de recherche de durabilité. Le cas de la Région de Bruxelles-Capitale, thèse de doctorat en art de bâtir et en urbanisme, université libre de Bruxelles.

NoËL Françoise, 2009, «La politique de revitalisation des quartiers : à la croisée de l'action urbanistique et sociale ", Bruxelles (dans) 20 ans, P. Dejemeppe, C. Mouchart, C. Piersotte, F. Raynaud et D. Van de Putte éd., Bruxelles, Région de Bruxelles Capitale, p. 213-233.

PUISSANT Jean, 2003, « Un siècle de logement social à Bruxelles », Le logement social au musée ?, M. Smets et P. Franqueville éd., Bruxelles, Luc Pire, p. 29-119.

RuMPALA Yannick, 2003, Régulation publique et environnement. Questions écologiques, réponses économiques, Paris, L'Harmattan.

VAN CRIEKINGEN Mathieu, 2006, "Que deviennent les quartiers centraux à Bruxelles ? »[en ligne], Brussels Studies, no 1, [URL : http://brussels.revues. org/293], consulté le 17 février 2017.

ZACCAI Edwin, 2011, 25 ans de développement durable, et après?, Paris, Presses universitaires de France.

ZÉLEM Marie-Christine, 2010, Politiques de maîtrise de la demande d'énergie et résistances au changement. Une approche socio-anthropologique, Paris, L'Harmattan. 
